\title{
Comparison of the prognosis of neoadjuvant chemoradiotherapy treatment with surgery alone in esophageal carcinoma: a meta-analysis
}

This article was published in the following Dove Press journal:

OncoTargets and Therapy

\author{
Hai-Feng Ma \\ Guo-Xiao Lv \\ Zhong-Fang Cai \\ Da-Hai Zhang
}

Department of Radiotherapy, Dongyang People's Hospital,

Dongyang, Zhejiang, China
Correspondence: Da-Hai Zhang Department of Radiotherapy, Dongyang People's Hospital, Number 60 West Wuning Road, Dongyang, Zhejiang 322100 , China

Tel +8657986859403

Email dyzdh@126.com
Background: Resection remains the best treatment for carcinoma of the esophagus in terms of local control, but local recurrence and distant metastasis remain an issue after surgery. Chemoradiotherapy (CRT) followed by surgery was associated with significantly improved survival benefit, but the effectiveness of neoadjuvant therapy in patients with resectable esophageal carcinoma remains controversial. The aim of this study was to evaluate the effects of neoadjuvant chemoradiotherapy in resectable esophageal carcinoma compared to surgery alone (SA).

Methods: A search for publications that compared the efficacy of CRT with SA in resectable esophageal carcinoma was conducted. After a rigorous review of the quality, the data were extracted from eligible trials. The major outcomes measures were odds ratios (ORs). The ORs with their corresponding $95 \%$ confidence intervals were the principal measure of effects. For the meta-analysis, Revman 5.3 software was used to analyze the combined pooled ORs using fixed- or random-effects models according to the heterogeneity.

Results: Our findings revealed that, compared with SA, neoadjuvant CRT was associated with improved overall survival (OS) and progression-free survival times, but the 3- and 5-year OS did not show a statistical difference $(P \geq 0.05)$. The adjuvant chemotherapy group did not show significant improvement on reference rate and metastasis rate compared with the control group.

Conclusion: CRT does significantly improve progression-free survival and OS in patients with esophageal cancer compared with SA. However, further assessment is still warranted on the role of CRT in future trials with well-selected patients.

Keywords: esophageal cancer, surgery, chemoradiation, neoadjuvant therapy

\section{Introduction}

Esophageal carcinoma is an aggressive malignancy of the gastrointestinal tract. Surgery has been the primary treatment for esophageal cancer; however, treatment failure results in many cases due to recurrence and distant metastases remain an issue after surgery.

Efforts have led to the investigation of multimodality therapies, and a combination of chemotherapy, radiotherapy, and surgery has been generally accepted as a reasonable option for patients with locoregional esophageal cancer. ${ }^{1,2}$ Radiotherapy can help with local disease control, while chemotherapy may be effective for both local and systemic antineoplastic activity.

Many studies have demonstrated the effectiveness of neoadjuvant CRT, which has been shown to lead to downsizing and downstaging the tumor and improving survival. ${ }^{3}$ However, toxic effects and compliance with protocols have hindered the development of multidisciplinary treatment. 
There is still controversy about how to improve prognosis and how to reduce local recurrence and distant metastasis. There has been limited information to suggest that either surgery or radiation/chemotherapy is a superior approach. This also raises the question about whether combining both would be superior to improve the postoperative quality of life and prolong the survival time. We performed this metaanalysis to assess the effect of neoadjuvant CRT on operable esophageal cancer compared to the surgery alone (SA).

\section{Methods and materials Search strategy}

Two investigators independently searched electronic databases PubMed, Embase, and Cochrane Library up to March 2017. The process was established to find all articles with the keywords: "esophageal neoplasm" AND "chemotherapy" "radiotherapy" AND “chemo-radiotherapy" AND "surgery", and relevant Medical Subject Heading terms were utilized. The reference lists of all articles that dealt with the topic of interest were also hand-searched to check for additional relevant publications.

\section{Eligibility criteria}

Studies were included in the meta-analysis if they met the following criteria: 1) trials evaluating CRT versus SA; 2) articles that provided data on the survival between patients from the CRT and those from the SA groups; 3) articles that described the cases and controls with regard to the reference $(R)$ rate and metastasis $(M)$ rate; and 4) studies that provided sufficient information to estimate the odds ratio (OR) and their 95\% confidence intervals (CIs). The studies not published in English were excluded.

\section{Quality assessment}

Two investigators independently rated the quality of the retrieved studies. We chose the risk of bias items recommended by The Cochrane Handbook for Systematic Reviews of Interventions as a quality indicator.

\section{Data extraction}

Two independent investigators extracted the relevant information from each study. Disagreement was revolved by consensus. From each of the eligible studies, the main categories that were extracted were as follows: first author family name, publication year, histology, treatment (size), endpoints of interest, and ORs with corresponding 95\% CIs or relevant data for OR and 95\% CI calculation for endpoints of interest.

\section{Statistical analysis}

The association between CRT and surgery with resected esophageal cancer is based on the data from trials. The endpoints of interest in the pooled analysis were 3-year overall survival (OS), 5-year OS, OS, progression-free survival (PFS), R data, and M data, and the endpoint outcomes were considered as a weighted average of individual estimate of the hazard ratio (HR) in every included study using the inverse variance method. If HRs and corresponding 95\% CIs were reported, lnHRs and the corresponding ln lower limits and $\ln$ upper limits were used as data points in pooling analysis.

A sensitivity analysis was also performed to examine the impact on the overall results, depending on the heterogeneity across the included studies. Heterogeneity was investigated by using the $I^{2}$ statistic. ${ }^{4}$ Studies with an $I^{2}$ of $25 \%-50 \%$, $50 \%-75 \%$, or $>75 \%$ were considered to have low, moderate, or high heterogeneity, respectively. ${ }^{5}$ Only if there was low heterogeneity among studies was the fixed-effects model used. Otherwise, the random-effects model was used. A $P$-value less than 0.05 was considered statistically significant. The statistical analyses were performed using Review Manager version 5.3 software (Revman; The Cochrane Collaboration, Oxford, UK). Findings of our meta-analysis are shown as forest plots.

\section{Results \\ Overview of literature search and study characteristics}

A total of 238 studies were retrieved initially for evaluation. Based on the criteria described in the methods, 10 publications were evaluated in more detail, but some did not provide data of outcomes of two approaches. Therefore, a final total of seven studies were included. ${ }^{6-12}$ The search process is described in Figure 1.

All included studies in this study were considered to be of moderate quality at least. Table 1 describes the primary characteristics of the eligible studies in more detail.

Clinical and methodological heterogeneity Pooled analysis of 3-year OS and 5-year OS between CRT and SA

Overall, six studies reported data on 3-year $\mathrm{OS}^{6-8,10-12}$ and four studies reported data on 5-year OS, ${ }^{7-9,12}$ and these are shown in Figures 2 and 3. Pooled data showed that CRT treatment did not show any benefit, with the pooled HR being 1.06 (95\% CI: $0.59-1.92, Z=0.21, P=0.83)$ and 0.91 (95\% CI: $0.44-1.89, Z=0.26, P=0.80$ ), respectively. 


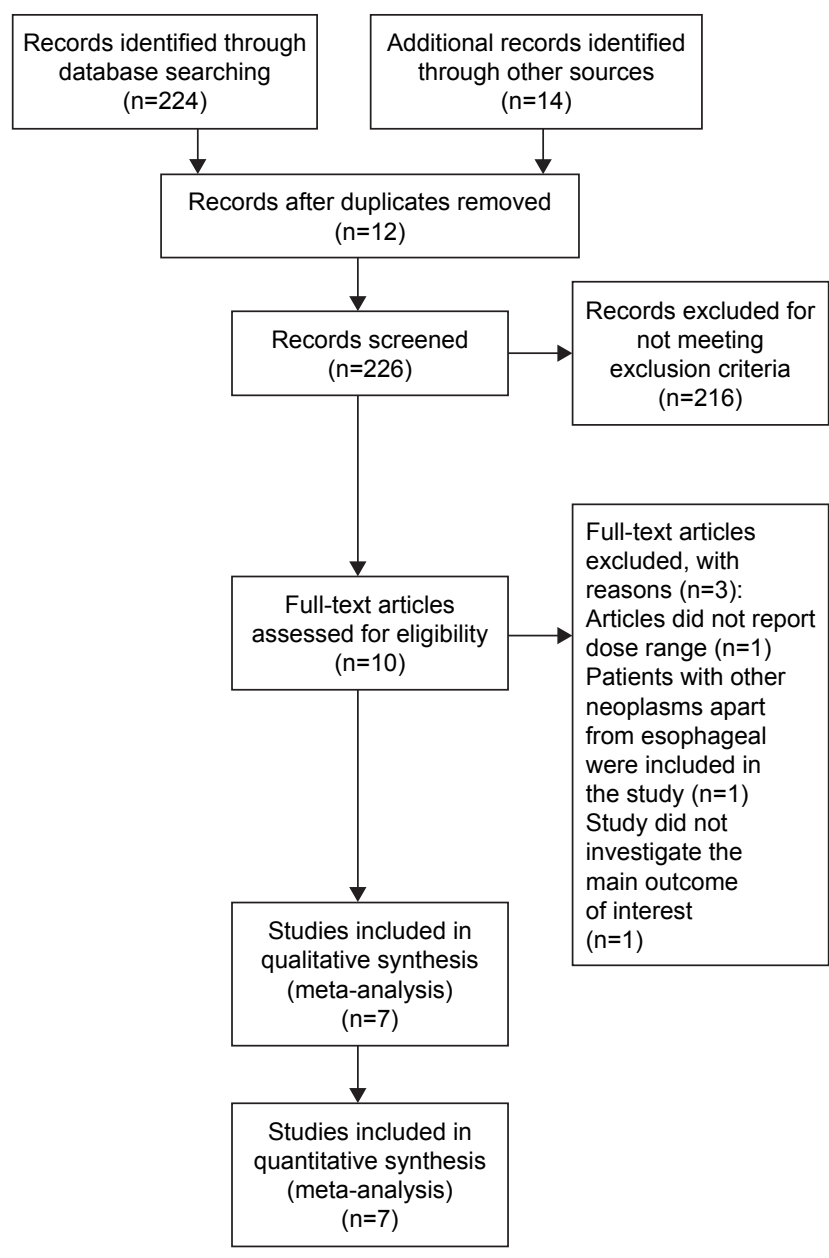

Figure I Preferred Reporting Items for Systematic Reviews and Meta-Analyses flow chart of selection process to identify studies eligible for pooling.

\section{Pooled analysis of OS between CRT and SA}

A total of seven studies ${ }^{6-12}$ reported the OS rates in patients (Figure 4). OS was superior in the CRT group compared with SA group (HR: $0.60,95 \%$ CI: $0.40-0.91, Z=2.40, P=0.02$ ).

\section{Pooled analysis of PFS between CRT and SA}

In the analysis of PFS in early-stage non-small-cell lung cancer, four studies ${ }^{7,8,11,12}$ were included that compared CRT with surgery. These data are shown in Figure 5. The PFS (HR: $0.36,95 \%$ CI: $0.16-0.79, Z=2.52, P=0.01$ ) benefits were seen in the CRT treatments.

\section{Pooled analysis of $R$ rate and $M$ rate between CRT and SA}

$\mathrm{R}$ rate and $\mathrm{M}$ rate were available for five trials $\mathrm{s}^{7,9,10-12}$ (Figure 6) and three trials, respectively ${ }^{10-12}$ (Figure 7). The aggregated results suggested that there was no R rate (HR: 0.35, 95\% CI: $0.10-1.19, Z=1.68, P=0.09$ ) or M rate (HR: $0.72,95 \%$ CI: $0.50-1.04, Z=1.73, P=0.08)$ benefit from CRT.

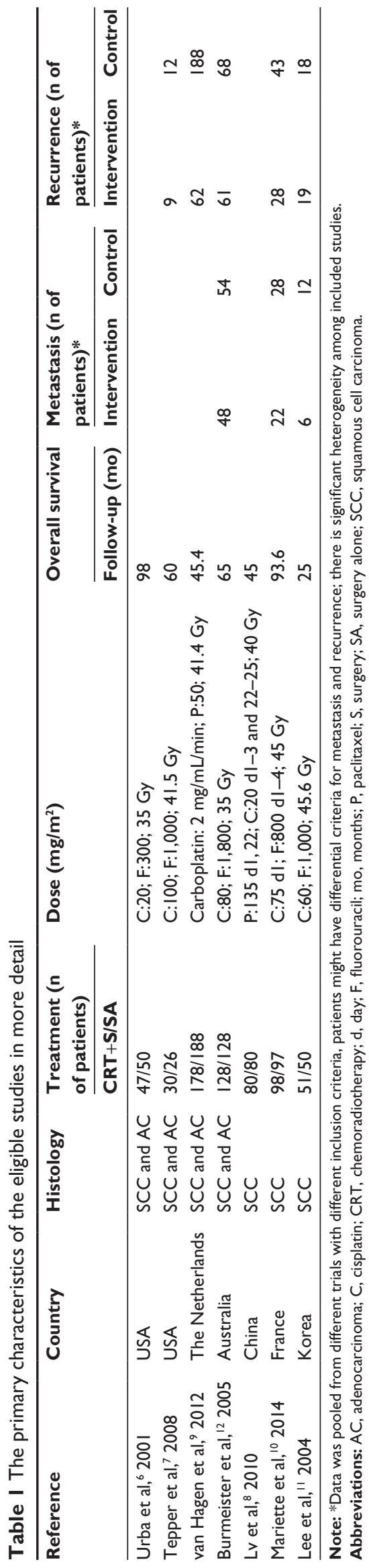




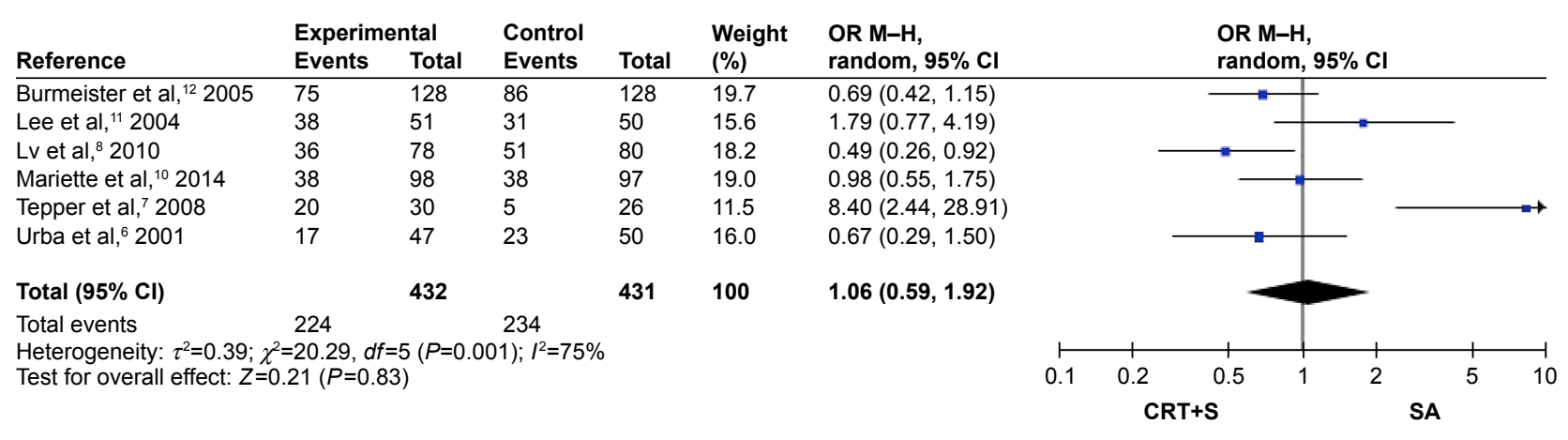

Figure 2 Pooled analysis of 3-year OS between CRT and SA.

Abbreviations: $\mathrm{Cl}$, confidence interval; CRT, chemoradiotherapy; M-H, Mantel-Haenszel; OR, odds ratio; OS, overall survival; S, surgery; SA, surgery alone.

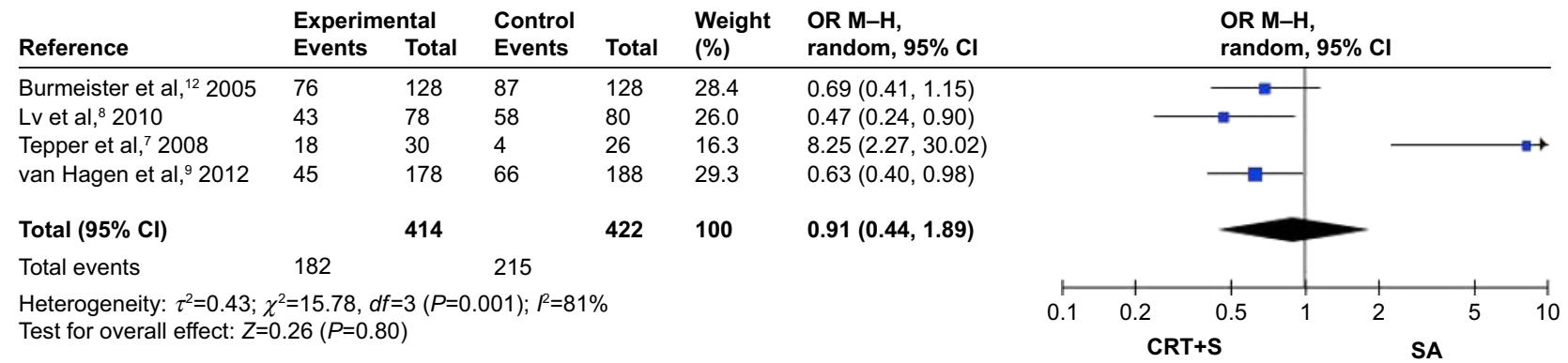

Figure 3 Pooled analysis of 5-year OS between CRT and SA.

Abbreviations: $\mathrm{Cl}$, confidence interval; CRT, chemoradiotherapy; $\mathrm{M}-\mathrm{H}$, Mantel-Haenszel; OR, odds ratio; OS, overall survival; S, surgery; SA, surgery alone.

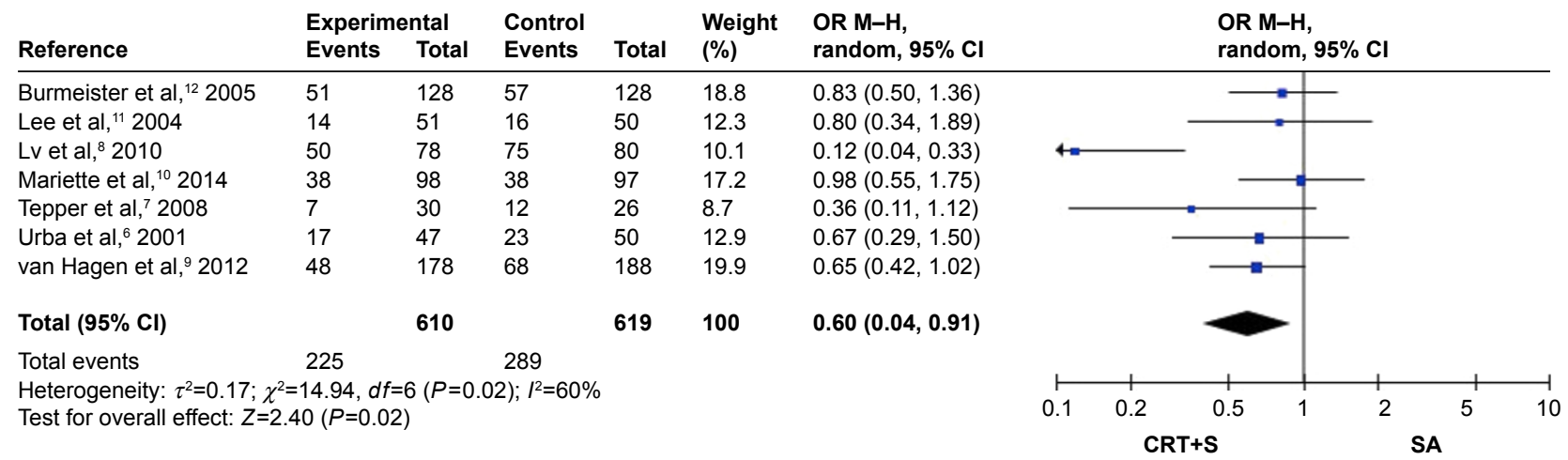

Figure 4 Pooled analysis of OS between CRT and SA.

Abbreviations: $\mathrm{Cl}$, confidence interval; CRT, chemoradiotherapy; M-H, Mantel-Haenszel; OR, odds ratio; OS, overall survival; S, surgery; SA, surgery alone.

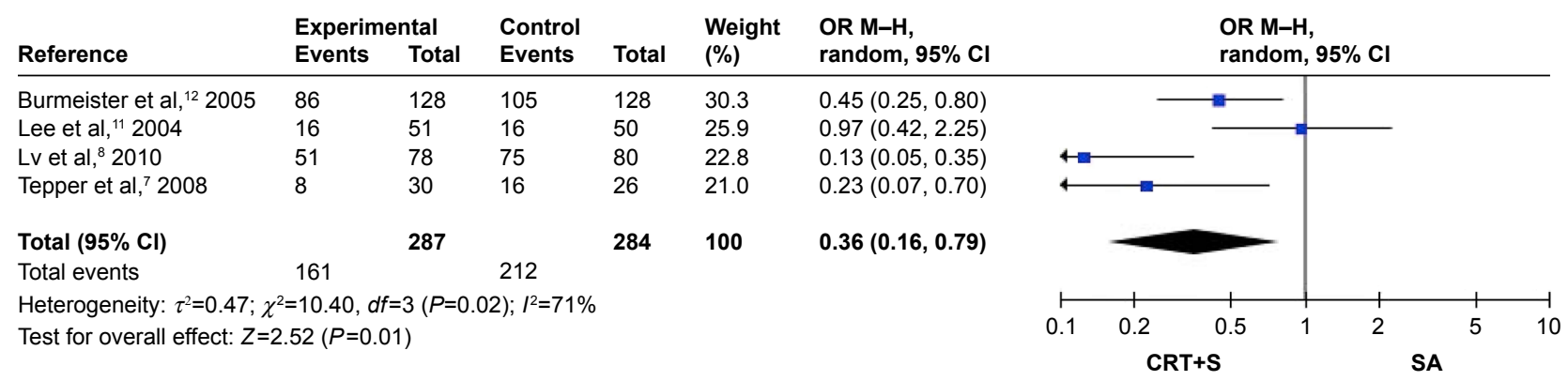

Figure 5 Pooled analysis of PFS between CRT and SA.

Abbreviations: $\mathrm{Cl}$, confidence interval; CRT, chemoradiotherapy; M-H, Mantel-Haenszel; OR, odds ratio; PFS, progression-free survival; S, surgery; SA, surgery alone. 


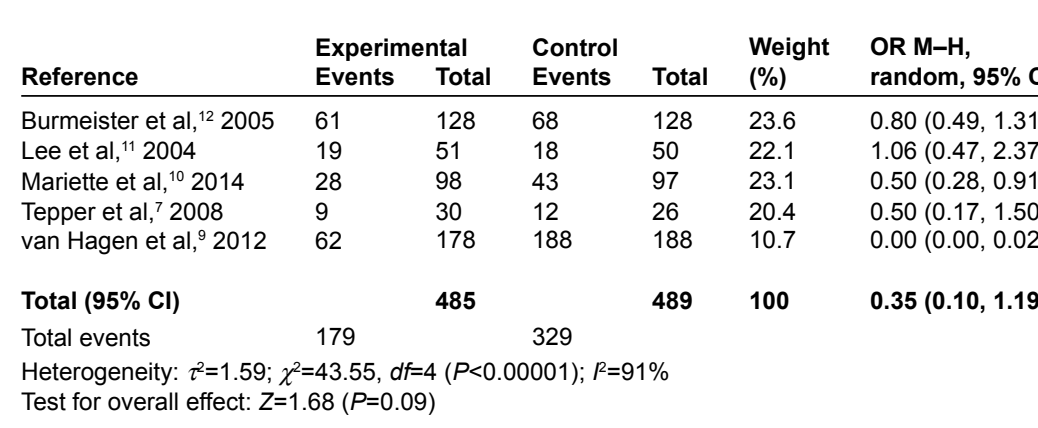

Figure 6 Pooled analysis of R rate between CRT and SA.

Abbreviations: $\mathrm{Cl}$, confidence interval; $\mathrm{CRT}$, chemoradiotherapy; $\mathrm{M-H}$, Mantel-Haenszel; OR, odds ratio; R, reference; S, surgery; SA, surgery alone.

\section{Discussion}

Surgery is potentially curative in locoregionally advanced cancer, but the morbidity and mortality associated with esophagectomy has restricted its role to a minority of medically fit patients. ${ }^{13}$ Local and distant recurrence dominates after surgical resection. ${ }^{14}$ Refinements in surgical technique have decreased postoperative mortality, but this has not been associated with prolonged OS. ${ }^{15}$

Lymph node recurrence was significantly lower in the CRT group than in the SA group. This result suggests that CRT was effective for local control.

However, a limited number of phase III trials on neoadjuvant CRT followed by surgery versus SA have produced conflicting results, contributing little to justify the routine use of CRT. ${ }^{16,17}$

A meta-analysis had suggested that preoperative CRT may improve survival and locoregional control but that it was associated with higher toxicity and increased mortality. Radiation might contribute to the failure of an anastomotic leak and postoperative acute lung injury. Long-term survival is maximized by the use of CRT followed by surgery for locally advanced esophageal cancer. However, patients are more likely to develop toxicity.

The rationale for the addition of irradiation to chemotherapy for resectable esophagectomy is based on good evidence of downstaging the tumor and improvement of local control, ${ }^{3}$ meaning that complete tumor resection is more probable and suboptimal surgery is less frequent. Evidently, such a downsizing effect is of greatest advantage in locally advanced tumors, where the integrity of the resection margin is more often threatened. There was a stronger benefit for better OS and PFS in the CRT-surgery arm compared with the surgery arm in this analysis. However, this study does not prove that neoadjuvant CRT improved the 3-year OS rate and 5-year OS rate, suggesting that differences in this demand further analysis.

CRT was effective for locoregional control. However, neither R rate nor $\mathrm{M}$ rate differed between groups $P=0.09$ and $P=0.08$, respectively. The reason for this lack of advantage in patients treated with neoadjuvant CRT can be explained in several ways. At first, there exists a publication bias for this meta might be ignored, the weaknesses of all included studies (underpowered different study design, doubtful staging accuracy and stratification, possible unbalanced randomization, variable radiation doses and its delivery, variable chemotherapy regimens and non-standardized surgical approach). In our study, the heterogeneity of the studies reporting available data on $\mathrm{R}$ rate was too high $\left(I^{2}=91 \%\right)$, so the true efficacy of trimodality therapy for esophageal cancer remains unclear and controversial. Second, pretrials show the effectiveness of the preoperative CRT regimen

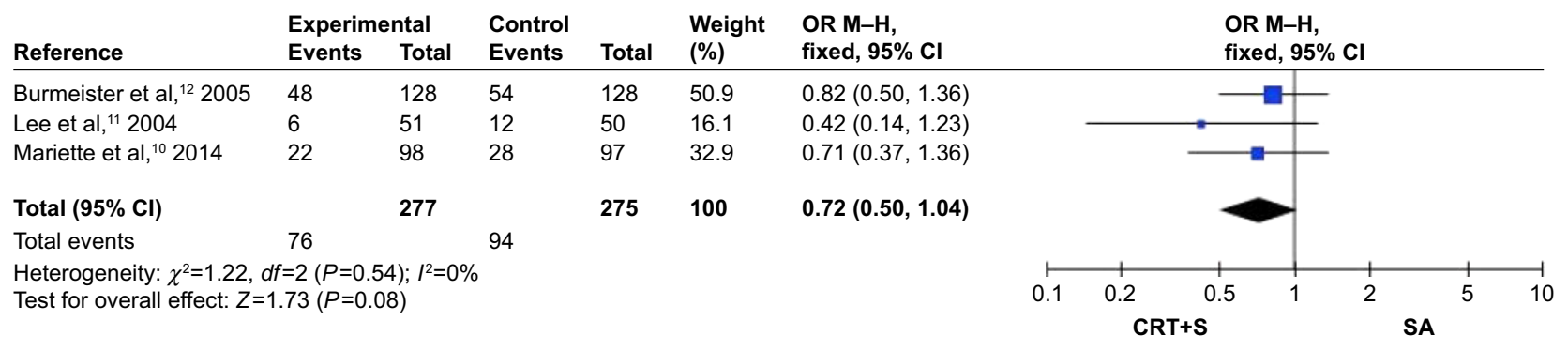

Figure 7 Pooled analysis of metastasis rate between CRT and SA.

Abbreviations: $\mathrm{Cl}$, confidence interval; $\mathrm{CRT}$, chemoradiotherapy; $\mathrm{M}-\mathrm{H}$, Mantel-Haenszel; OR, odds ratio; S, surgery; SA, surgery alone. 
in patients with squamous cell cancer, and the outcome is more encouraging. ${ }^{18}$ The doses used in our regimen might be adequate for a benefit in patients with squamous cell carcinoma; however, we cannot conclude this possibility with certainty on the basis of the subgroup analysis. So the different responses of squamous cell cancers and nonsquamous cell cancers to CRT may be a prognostic factor for survival owing to the difference in histology of the tumor type, and this should be assessed separately in future. Moreover, many studies have paid attention to molecular markers, such as epidermal growth factor receptor inhibitors ${ }^{19}$ and cycloxygenase- $2 .{ }^{20}$ The expression level of several genes changed after neoadjuvant therapy. For example, thymidine synthesis, dihydropyrimidine dehydrogenase, glutathione S-transferase Pi, epidermal growth factor receptor, and human epidermal growth factor receptor 2 are negatively expressed. ${ }^{21}$ Thus, whether or not the survival benefit of neoadjuvant CRT can be negated by molecular markers should be taken into consideration.

The possible benefit of neoadjuvant CRT would necessarily depend on discriminating the patients qualified and not qualified to benefit from the neoadjuvant therapy and on preventing unnecessary harm to and reducing the therapeutic expense of the treatment. As treatment regimens improve and the incidence of detection of earlier-stage disease increases, ${ }^{14}$ improved patient compliance rates mandate an alternative approach to patients who cannot benefit from resection but are placed at risk for mortality and long-term morbidity from surgery.

A possible limitation of this study is its nonrandomized nature. We did not observe more survival differences between two groups from the unmatched analysis. The translation of outcomes into a survival benefit might be more pronounced with well-selected patients.

\section{Conclusion}

Further efforts for individualized therapy for CRT should be considered according to multiple aspects such as pathologic type of the tumor ${ }^{22}$ and the biological and molecular markers of the tumor in order to maximize the benefit and minimize the harmful effects of neoadjuvant CRT. As therapies improve, it is likely that the toxicity may be reduced and neoadjuvant treatment may provide a more marked benefit in esophageal cancer.

\section{Disclosure}

The authors report no conflicts of interest in this work.

\section{References}

1. Di Fiore F, Lecleire S, Rigal O, Galais MP. Predictive factors of survival in patients treated with definitive radiochemotherapy for squamous cell esophageal carcinoma. World J Gastroenterol. 2006;12:4185-4190.

2. Li S, Liu H, Diao C, et al. Prognosis of surgery combined with different adjuvant therapies in esophageal cancer treatment: a network meta-analysis. Oncotarget. 2017;8(22):36339-36353.

3. Sjoquist KM, Burmeister BH, Smithers BM, et al. Survival after neoadjuvant chemotherapy or chemoradiotherapy for resectable oesophageal carcinoma: an updated meta-analysis. Lancet Oncol. 2011;12:681-692.

4. Higgins JP, Thompson SG. Quantifying heterogeneity in a metaanalysis. Stat Med. 2002;21(11):1539-1558.

5. Higgins JP, Thompson SG, Deeks JJ, Altman DG. Measuring inconsistency in meta-analyses. BMJ. 2003;327(7414):557-560.

6. Urba SG, Orringer MB, Turrisi A, Iannettoni M, Forastiere A, Strawderman M. Randomized trial of preoperative chemoradiation versus surgery alone in patients with locoregional esophageal carcinoma. J Clin Oncol. 2001;19(2):305-313.

7. Tepper J, Krasna MJ, Niedzwiecki D, et al. Phase III trial of trimodality therapy with cisplatin, fluorouracil, radiotherapy, and surgery compared with surgery alone for esophageal cancer: CALGB 9781. J Clin Oncol. 2008;26(7):1086-1092.

8. Lv J, Cao XF, Zhu B, Ji L, Tao L, Wang DD. Long-term efficacy of perioperative chemoradiotherapy on esophageal squamous cell carcinoma. World J Gastroenterol. 2010;16(13):1649-1654.

9. van Hagen P, Hulshof MC, van Lanschot JJ, et al. Preoperative chemoradiotherapy for esophageal or junctional cancer. $N$ Engl J Med. 2012; 366(22):2074-2084.

10. Mariette C, Dahan L, Mornex F, et al. Surgery alone versus chemoradiotherapy followed by surgery for stage I and II esophageal cancer: final analysis of randomized controlled phase III trial FFCD 9901. J Clin Oncol. 2014;32(23):2416-2422.

11. Lee JL, Park SI, Kim SB, et al. A single institutional phase III trial of preoperative chemotherapy with hyperfractionation radiotherapy plus surgery versus surgery alone for resectable esophageal squamous cell carcinoma. Ann Oncol. 2004;15(6):947-954.

12. Burmeister BH, Smithers BM, Gebski V, et al. Surgery alone versus chemoradiotherapy followed by surgery for resectable cancer of the oesophagus: a randomised controlled phase III trial. Lancet Oncol. 2005; 6(9):659-668.

13. Dubecz A, Gall I, Solymosi N, et al. Temporal trends in long-term survival and cure rates in oesophageal cancer: a SEER database analysis. J Thorac Oncol. 2012;7(2):443-447.

14. Edgren G, Adami HO, Weiderpass Vainio E, Nyren O. A global assessment of the oesophageal adenocarcinoma epidemic. Gut. 2013;62(10): 1406-1414.

15. Barnett SA, Rizk NP. Randomized clinical trials in oesophageal carcinoma. Surg Oncol Clin N Am. 2010;19(1):59-80.

16. Urba SG, Orringer MB, Turrisi A, et al. A randomized trial of preoperative chemoradiation versus surgery alone in patients with locoregional esophageal cancer. J Clin Oncol. 2000;19:303-313.

17. Burmeister BH, Smithers BM, Fitzgerald L, et al. A randomized phase III trial of preoperative chemoradiation followed by surgery versus surgery alone for localized resectable cancer of the esophagus [abstract]. Proc Am Soc Clin Oncol. 2002;21:130a.

18. Rizk NP, Seshan VE, Bains MS, et al. Prognostic factors after combined modality treatment of squamous cell carcinoma of the oesophagus. J Thorac Oncol. 2007;2(12):1117-1123.

19. Trial register NCT01107639; 2013. Available from: http://www. clinicaltrials.gov/ct2/show/NCT01107639?term=ruhstaller\&rank=1. Accessed January 31, 2013.

20. Xi H, Baldus SE, Warnecke-Eberz U, et al. High cyclooxygenase-2 expression following neoadjuvant radio-chemotherapy is associated with minor histopathologic response and poor prognosis in esophageal cancer. Clin Cancer Res. 2005;23:8341-8347. 
21. Schneider S, Uchida K, Brabender J, et al. Downregulation of TS, DPD, ERCC1, GST-Pi, EGFR, and HER2 gene expression after neoadjuvant three-modality treatment in patients with esophageal cancer. $J$ Am Coll Surg. 2005;3:336-344.
22. Lambin P, van Stiphout RG, Starmans MH, et al. Predicting outcomes in radiation oncology - multifactorial decision support systems. Nat Rev Clin Oncol. 2012;10:27-40.

\section{Publish your work in this journal}

OncoTargets and Therapy is an international, peer-reviewed, open access journal focusing on the pathological basis of all cancers, potential targets for therapy and treatment protocols employed to improve the management of cancer patients. The journal also focuses on the impact of management programs and new therapeutic agents and protocols on

\section{Dovepress}

patient perspectives such as quality of life, adherence and satisfaction. The manuscript management system is completely online and includes a very quick and fair peer-review system, which is all easy to use. Visit http://www.dovepress.com/testimonials.php to read real quotes from published authors.

Submit your manuscript here: http://www.dovepress.com/oncotargets-and-therapy-journal 